\title{
FATORES PROGNÓSTICOS E PREDISPOSIÇÃO GENÉTICA ASSOCIADA AO CÂNCER DE MAMA
}

\author{
Alanna Evangelista dos Santos ${ }^{1}$ \\ Felicson Leonardo Oliveira Lima ${ }^{2}$ \\ 1-Graduanda em Biomedicina pela Unidade de Ensino Superior de Feira de Santana; \\ 2-Biomédico; Especialista em Biologia Celular e Molecular; Mestrando em \\ Biotecnologia pela Universidade Estadual de Feira de Santana; \\ E-mail para correspondência: alannaevangelistasantos@outlook.com
}

\begin{abstract}
RESUMO
O câncer de mama é a segunda neoplasia mais frequente no mundo, demonstrando prevalência e mortalidade maiores sob o sexo feminino, acometendo, porém, em uma menor escala o sexo masculino. A identificação precoce dos fatores de prognóstico, bem como o entendimento, frente a predisposição genética, permitirá ao indivíduo acometido maiores chances de sobrevida. Para tanto, o presente estudo, enquadra-se como uma revisão bibliográfica com abordagem narrativa e de caráter qualitativo, onde objetivouse abordar os principais fatores prognósticos e a preposição genética associada à herança da mutação no câncer de mama. Pode-se concluir através deste estudo que o fator de prognóstico é preditor na sobrevida do paciente, e que as correlações genéticas de mutação nos genes BRCA1 e BRCA2 aumentam as chances do câncer de mama por hereditariedade.
\end{abstract}

Palavras-chave: Câncer de mama; Neoplasia da mama; Predisposição Genética; Prognóstico. 


\section{INTRODUÇÃO}

O câncer de mama é uma patologia que consiste em alterações nos mecanismos celulares, promovendo modificações nos processos do ciclo celular, sendo a proliferação e a apoptose alteradas cromossomicamente. Ocorrendo assim, a ativações de oncogênese e a ineficiência de genes de supressores tumorais (BATSCHAUER, 2009).

Sabe-se que o câncer de mama é a segunda neoplasia mais frequente no mundo. Além de ser mais comum em mulheres, representa a principal causa de morte das brasileiras, perdendo somente para o câncer de pulmão (CANTINELLI et al., 2006).

Sendo considerado um problema de saúde pública em todo mundo, a alta taxa de incidência do câncer de mama requer uma maior prevenção, diagnóstico e controle da doença. Nos países desenvolvidos que apresentam as medidas supracitadas a taxa de sobrevida é de $61 \%$ após cindo anos de descoberta do câncer (BRASIL, 2009).

Raramente o câncer de mama se desenvolve antes dos 35 anos, crescendo, porém, progressivamente com a idade. Normalmente as mulheres descobrem a doença entre 40 e 60 anos, em contrapartida, na atualidade, a doença está atingindo um maior número de mulheres, estimando uma incidência de 43,74\% para 2021 (INCA, 2020).

Apesar do câncer de mama ser considerado uma doença multifatorial, os riscos envolvem fatores internos, como a constituição hormonal ou a predisposição hereditária, e fatores externos como agentes químicos ou biológicos estilo de vida, excesso de peso, sedentarismo, radiação ionizante e consumo de álcool (BRASIL, 2009).

Neste sentido, a hereditariedade é um dos fatores mais predominante, pois aproximadamente $10 \%$ da incidência de câncer de mama estão relacionados à mutação genética e aconteceram em indivíduos com histórico familiar de câncer de mama (INCA, 2021). 
Por se tratar de uma doença com grande impacto psicológico, emocional e social, além de sua grande relevância para o estudo de sua fisiopatologia, diagnóstico e tratamento, o objetivo desde estudo foi de abordar os principais fatores prognósticos e a preposição genética associada à herança da mutação no câncer de mama.

\section{METODOLOGIA}

Trata-se de um estudo de revisão bibliográfica com abordagem narrativa e de caráter qualitativo, onde foram analisados artigos sobre a temática nas bases de dados: Biblioteca Virtual de Saúde (BVS), SciELO, PubMed e LILACS, publicados entre os anos de 2005 a 2021, excedendo-se apenas de um estudo de extrema importância, publicado em 1989.

Através do filtro avançado das fontes supracitadas, foram selecionados os estudos indexados seguindo os determinados critérios de inclusão e exclusão. As palavras-chave foram utilizadas os seguintes termos: Câncer de mama; Neoplasia da mama; Predisposição Genética; Prognóstico.

Os critérios de inclusão foram empregados em estudos publicados entre os anos de 2005 a 2021, que abordasse especificamente os objetivos do presente estudo, materiais publicados em inglês ou português, com teor de originalidade. Para os critérios de exclusão, foram descartados materiais duplicados, publicados fora do recorte temporal e incompletos.

\section{RESULTADOS E DISCUSSÕES}

Para Pinheiro (2013), o fator com maior teor de preocupação para população feminina é o câncer de mama, é a neoplasia que causa um grande impacto psicológico, social e físico no doente. Ele atua de forma negativa em relação com a imagem pessoal, aumentando a insatisfação com a percepção no que tange a sexualidade do paciente. Martins (2001), por sua vez, retratou em seu estudo que diversos são os fatores que causam o câncer de mama. Todavia, 
quando um fator pode ser definido no momento do diagnóstico, o mesmo é denominado fator prognóstico, pois o mesmo serve como preditor da sobrevida ou do tempo livre que o paciente tem da doença.

Conhecer o fator prognóstico permite um maior avanço da ciência. Os estudos científicos tem focado cada dia mais na apresentação destes fatores, porém na prática ainda não é tão factível de serem consideradas, devido diversas circunstâncias para sua definição (DONEGAN, 2001).

Quanto maior o número de fatores determinado no prognóstico, maior o número de possíveis associações a serem realizadas, permitindo que os preditores sejam identificados e forneçam informações da provável recidiva do tumor de mama (URBAN, et al., 2012).

O tamanho do tumor e as condições dos linfonodos auxiliares são dois dos mais importantes prognósticos, e podem ser identificados na mamografia, utilizada como exame de prevenção, por meio de campanhas que visam conscientizar a população sobre a sua importância. Estudos comprovam que para pacientes diagnosticados precocemente com tumores menores do que $2 \mathrm{~cm}$ apresentam uma sobrevida de $88 \%$ (SILVA et al., 2011).

A presença de linfonodos auxiliares ocorre frequentemente, este é conhecido como metástase, e seu risco são diretamente proporcionais ao seu tamanho, ou seja, tumores acima e $1 \mathrm{~cm}$ apresentam aproximadamente $30 \%$ de chance de serem tumores ductais com grau de comprometimento elevado (NICO et al., 2016).

A classe social, também é um fator prognóstico a ser considerado. As classes menos favorecidas são prejudicadas, pois em grande parte das vezes descobrem a doença já em fase avançada (BATSCHAUER, 2009).

O grau histológico é um fator responsável pela reflexão de potencialidades de malignidade, pois indica a sua capacidade de metastifiscação, mas a sua 
natureza ainda é tida como subjetiva entre as diferentes patologias gerando controvérsias e gerando dificuldades na sua utilização (PINHEIRO, 2013).

A idade também influência no momento do diagnóstico, existindo preponderância de um melhor prognóstico em pacientes jovens com até 35 anos e também após os 75 anos de idade. Este fator se mostrou tendencioso em idades de 45 a 49 anos em estudos já realizados (STAFIN et al., 2012).

Sabe-se que existem diversos fatores prognósticos pesquisados que podem contribuir para existência do câncer de mama, os supracitados neste estudo são uma exemplificação do quanto à ciência está e ainda pode avançar nesta área.

Ainda neste sentido, considerando a doença como multifatorial, este estudo propõe discutir sobre a hereditariedade como principal fator epidemiológico para o desenvolvimento de câncer de mama e suas mutações (PINHEIRO, 2013).

Este está diretamente relacionado ao agrupamento familiar de síndromes com características próprias, que acabam conferindo um risco elevado ao parente da pessoa com câncer, do que ao restante da população (MAVADDAT, 2010).

Estudos anteriores relatam que mutações em genes supressores de tumor como BRCA1 e BRCA2, contribuem para o aumento do risco de câncer de ovário e do câncer de mama. Assim, as famílias em que apresentavam várias pessoas com câncer de mama, tinham 52\% de mutação no BRCA1 e 32\% no BRAC2 (CESAR, 2012).

Desta forma, é necessária a identificação de casos de câncer hereditários na família, pois assim o rastreamento intensivo tende a reduzir o risco de mutação e permite que o indivíduo escolha utilizar medidas de profilaxias como cirurgias profiláticas e quimioprofilaxia (MAVADDAT, 2010).

Por fim, o diagnóstico clínico hereditário é definido pelas bases fundamentais da NCCN (National Comprehensive Cancer Network) e SCO (American Society of Clinical Oncology), No campo da saúde, uma variedade de ferramentas foram desenvolvidas para avaliar Distress, algumas das quais têm sido utilizadas em pesquisas com pacientes Oncológicos, denominado (Distress Management), sendo um manual elaborado por 
profissionais de diferentes áreas relacionadas à atenção ao câncer, que objetiva estabelecer quadros de sintomas e sugerir métodos de intervenção e monitoramento, além de garantir que medidas preventivas sejam tomadas para reduzir ou eliminar possíveis transtornos mentais (DECAT et al., 2009)

\section{CONSIDERAÇÕES FINAIS}

Mediante informações dos estudos explanados, pôde-se elucidar os principais fatores prognósticos preditores da sobrevida do paciente com câncer de mama, sendo definidos no momento do diagnóstico. Ainda assim, estas pesquisas apontam a hereditariedade como um fator importante no desenvolvimento do câncer de mama, que ocorrem através da mutação dos genes BRC1 e BRCA2. É necessário conscientizar a população sobre a necessidade de utilização dos mecanismos de diagnóstico e prevenção como a mamografia. Mais estudos devem ser realizados sobre os impactos físicos, psicológicos e sociais em um indivíduo com câncer de mama. 


\section{REFERÊNCIAS}

BATSCHAUER, A. P. B. Avaliação hemostática e molecular em mulheres com câncer de mama receptor hormonal negativo. 2009. Tese (Doutorado em Ciências Farmacêuticas). Faculdade de Farmácia, Universidade Federal de Minas Gerais, Belo Horizonte, 2009.

CANTINELli FS, CAMACHO RS, SMALETZ O, GONSALES BK, Braguittoni E, Rennó JR. A oncopsiquiatria no câncer de mama: considerações a respeito de questões do feminino. Rev Psiquiatr Clín 2006;33(3):124-33

Cesar PGC, Fonseca FLA, Gehrke FS, Alves BCA, Kuniyoshi RK, Giglio AD. Utilização de plataforma gênica no prognóstico do câncer de mama. Arq Bras Ciên Saúde. 2012;17(13):156-58.

DECAT, Cristiane Sant'Anna; LAROS, Jacob Arie; ARAUJO, Tereza Cristina Cavalcanti Ferreira de. Termômetro de distress: validação de um instrumento breve para avaliação diagnóstica de pacientes oncológicos. Psico-Usf, v. 14, p. 253-260, 2009.

HAYBITTLE, J. L. et al. A prognostic index in primary breast cancer. British journal of cancer, v. 45, n. 3, p. 361-366, 1982.

Instituto Nacional de Câncer José Alencar Gomes da Silva. Rede Nacional de Câncer Familial - Manual Operacional. [Internet] Rio de Janeiro: Inca; [acesso em 2016 Jul 15]. Disponível em: http://bvsms.saude. gov.br/bvs/publicacoes/rede_nacional_cancer_manual.pdf.

Instituto Nacional De Câncer José Alencar Gomes Da Silva. Atlas da mortalidade. Rio de Janeiro: INCA, 2021. 1 base de dados. Disponível em: https://www.inca.gov.br/app/mortalidade Acesso em: 07 ago 2021

MARTINS, Luhan Chaveiro et al. Padrão de metástase no câncer de mama triplo negativo. Rev Bras Mastologia, v. 27, n. 1, p. 8-14, 2017.

MAVADDAT N, ANTONIOU AC, EASTON DF, GARCIA-CLOSAS M. Genetic susceptibility to breast cancer. Mol Oncol. 2010 Jun;4(3):174-91

Ministério da Saúde (BR). Instituto Nacional do Câncer. Estimativas 2010: incidência de câncer no Brasil. Rio de Janeiro: INCA; 2009.

NICO, João Douglas et al. ESTADIAMENTO DO CÂNCER DE MAMA EM MULHERES ATENDIDAS EM UM CENTRO DE REFERÊNCIA EM ONCOLOGIA. Arquivos de Ciências da Saúde da UNIPAR, v. 20, n. 2, 2016. 
PINHEIRO AB, LAUTER DS, MEDEIROS GC, CARDOZO IR, MENEZES LM, BARRETO RM, ET AL. Câncer de Mama em Mulheres Jovens: Análise de 12.689 casos. Rev Bras Cancerol. 2013;59(3):351-9.

ROSEN PP, GROSHEN S, SAIGO PE, KINNE DW, Hellman S. Pathological prognostic factors in stage I (T1N0M0) and stage II (T1N1M0) breast carcinoma: a study of 644 patients with median follow-up of 18 years. J Clin Oncol 1989;7:1239-45.

SCLOWITZ ML, MENEZES AMB, GIGANTE DP, TESSARO S. Condutas na prevenção secundária do câncer de mama e fatores associados. Rev Saúde Pública 2005;39(3):340-9.

SILVA, Pamella Araújo da; RIUL, Sueli da Silva. Câncer de mama: fatores de risco e detecção precoce. Revista Brasileira de Enfermagem, v. 64, p. 1016-1021, 2011.

STAFIN, Inês et al. Fatores prognósticos no câncer de mama. HU Revista, v. 38, n. 3 e 4, 2012.

URBAN, Linei Augusta Brolini Dellê et al. Recomendações do Colégio Brasileiro de Radiologia e Diagnóstico por Imagem, da Sociedade Brasileira de Mastologia e da Federação Brasileira das Associações de Ginecologia e Obstetrícia para rastreamento do câncer de mama por métodos de imagem. Radiologia brasileira, v. 45, p. 334-339, 2012. 